\title{
Über die Furchungserscheinung am Ei des Tritons im Aktionsstrombild.
}

\author{
Von \\ Kiyoshi Kikuchi. \\ Aus dem Anatomischen Institut der Medizinischen Fakultät \%u Nagasaki \\ (Direktor: Prof. Dr. K. Kunitomo).
}

Mit 1 Textabbildung.

Bei der embryologischen Untersuchung des Amphibieneies ist die Aufmerksamkeit der Autoren bisher hauptsächlich nur auf die morphologischen Veränderungen ${ }^{1)}$ gerichtet gewesen. Wenn man andererseits mit irgendeiner Methode den Energieumsatz des Eies bei seiner Entwicklumg beobachten könnte, so liesse sich vielleicht der Embryologie ein neues Forschungsfeld eröffnen. Dies scheint möglich durch die Beobachtung der Aktionsströme an der Eioberfläche. Es liegt die Annahme nahe, dass man bei Furchung des Tritoneies von seiner Oberfläche Potentialschwankungen ableiten kömnte, weil dabei anscheinend ein nicht geringer Energieumsatz vor sich geht. Jies ist aber trotz der geistreichen Idee bisher vernachlässigt worden ${ }^{233}$.

Das Ei des Tritons ist zu diesem Zweck besonders geeignet, weil es ziemlich gross ist und man daher das zeitliche Verhalten des Furchungsprozesses gut beobachten kann.

\section{Methodik.}

Zur Laichzeit habe ich Männchen und Weibchen des Tritons aus Tümpeln oder Teichen gefangen und im Aquarium des Instituts gehalten. Dabei geschah die Eiablage wie normal. Nach der Eiablage pflegte das 
Männchen über den soeben abgelegten Eiern hin- und herzuschwimmen. Dabei gewann man den Eindruck, als ob es seinen Samen ejakulierte.

Die Eier wurden vor ihrer äusserer Eihülle und Gallertmasse blossgelegt, wobei die innere Eihülle, nämlich die Befruchtungsmembran, dem Eidotter anhaften blieb. Das so behandelte Ei wurde auf das in einer Glasplatte angebrachte kleine Loch gelegt. Die Ableitung der Aktionsströme geschah durch zwei Nadelelektroden-Stahlnähnadeln. Die Spitze der einen Nadel berührte den animalen Pol und die der anderen, von unten durch das Loch der Glasplatte hindurch, auf die das Ei gelegt war, den vegetativen-diphasische Ableitung. Wenn man das Ei auf der Glasplatte dauernd mit Wasser befeuchtete, so setzte die Furchungserscheinung sich fort. Manchmal aber bei langem Aufliegen des Eies auf der Glasplatte komnte die Furchung durch Trocknung mehr oder weniger behindert werden; die Aktionsströme der einzelnen Furchungsstadien wurden daher von verschiedenen Eiern abgeleitet.

Der Galvanometer (Cambridge-Modell) wurde mit den Elektroden so verbunden, dass die Negativität des animalen Pols Saitenausschlag nach oben gab. Die Empfindlichkeit der Saite betrug bei Einführung der Elektroden und des Eies in den Stromkreis und bei 1 Meter Registrierabstand und 1000 facher optischer Vergrösserung $7 \mathrm{~cm}$ für 1 millivolt. Bei photographischer Aufnahme des Aktionsstrombildes fiel die Trockenplatte in der Kamera automatisch nach unten. Hier babe ich der Bequemlichkeit halber die Ableitung der Aktionsströme in den früheren 3 Furchungsstadien vorgenommen.

\section{Versuchsergebnisse.}

1. Bei der ersten Furchung.

Bei befruchtetem Ei geschahen schon vor der ersten Furchung stundenlang ab und zu leichte Potentialschwankungen (mono- oder diphasische), vielleicht als elektromotorischer Ausdruck des Bildungsprozesses der Furchungsspindel (Vereinigung der beiden Vorkerme), denn der animale Pol, wo die in Aktion gesetzten Kerne enthalten sind, ist gegenüber dem ruhigen vegetativen Pol immer elektronegativ.

Bei Wassertemperatur von $8-12^{\circ} \mathrm{C}$ begann die erste Furchung. Etwa 1 Stunde vor Beginn derselben nahmen die Potentialschwankungen besonders zu, wobei die Form der Schwankungen rhythmisch mono- oder diphasisch war. Dabei war der animale Pol gegenüber dem vegetativen immer elektronegativ; die Ableitung der Elektrophänomene war von den

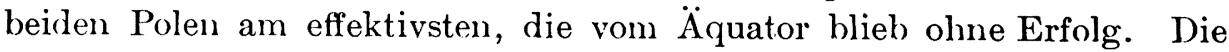


Potentialschwankungen dauerten etwa $1 / 2$ Stunde fort und pflegten etwa 1/2 Stunde vor Beginn der ersten Furchung zu verschwinden. Diese elektrischen Schwankungen könnte man vielleicht auf die Aktionsströme der ersten Kernteilung, welche die erste Furchung der Fioberfläche vorbereitet, zurückführen. Dafür sprechen die histologischen Befunde von manchen Autoren, dass der Furchungskern schon einige Zeit vor der Furchung der Eioberfläche seinen Teilungsvorgang beendet hat. Die Furchung der Eioberfläche geschah in der animalen Hälfte ziemlich schnell (15-20'), damn aber in der vegetativen Hälfte langsam (2-3 Stunden). Mit Beginn der ersten Furchung konnte man rhythmisch monooder diphasische Elektrophänomene von der Eioberfläche ableiten, wobei der animale $\mathrm{Pol}$ gegenüber dem vegetativen elektronegativ wurde. Die Ableitung vom Äquator blieb ohne Effekt.

Entsprechend der Geschwindigkeit der Furchung der Eioberfläche waren die Potentialschwankungen zuerst stark und frequent; bei den späteren Stadien d. h. bei der Furchenbildung in der vegetativen Hälfte waren die Schwankungen langsam und schwach. Die besagten Potentialschwankungen sind zweifellos Aktionsströme infolge der Furchenbildung im Eidotter oder im Protoplasma.

Bei Furchung in der vegetativen Hälfte war der vegetative Pol gegenüber dem animalen elektronegativ. Die Potentialschwankung bei Furchung in der animalen Hälfte war grösser und schmeller als die bei Furchung in der vegetativen Hälfte. Die Potentialdifferenz vor der I. Furchung war etwas grösser als während derselben. Etwa-1 Stunde vor der II. Furchung-wobei die I. Furchung schon den vegetativen Pol erreicht hatte-traten wieder Potentialschwankungen auf, die einige Zeit andauerten und etwa 20-30 Minuten vor der II. Furchung zu verschwinden pflegten. Dabei wurde der animale Pol gegenüber dem vegetativen elektronegativ. Auf Grund des von manchen Autoren angegebenen histologischen Befundes, dass vor den einzelnen Furchungen die entsprechende Kernteilung schon beendet ist, sind diese Potentialschwankungen auf die der II. Furchung entsprechende II. Kernteilung zurückführbar.

2. Bei der II. Furchung.

Die II. Furchung, die etwa 1-2 $\frac{x}{2}$ Stunden nach Beginn der I. Furchung begann, geschah auch vertikal, wobei die I. und II. Furchung sich rechtwinklig kreuzten. Die Furchenbildung in der animalen Hemisphäre geschah ziemlich schnell $\left(20-25^{\prime}\right)$, in der vegetativen Hemisphäre aber langsam (1/2-2 Stunden). Mit Beginn der II. Furchenbildung beobachtete ich wieder Potentialschwankungen-der animale Pol 
wurde elektronegativ gegenüber dem vegetativen. Dabei war die Ableitung von den beiden Polen am effektivsten; erfolglos aber war Ableitung vom Äquator. Diese Potentialschwankungen beruhen zweifellos auf dem Teilungsprozess des Eidotters und Protoplasmas. Die Potentialdifferenz bei Teilung des Eidotters und Protoplasmas war grösser und schneller als die bei Kernteilung. Die Potentialdifferenz bei der I. Furchung war schwächer als die bei der II.-ten. Bei der II. Furchung in der animalen. Hemisphäre wurde der vegretative Pol elektronegativ gegenüber dem animalen.

Zu der Zeit, wo die II. Furchung schon den vegetativen Pol erreicht hatte und die Potentialschwankungen auch schon beendet war, geschahen wieder plötzlich Potentialschwankungen, was gewöhnlich etwa 1 Stunde nach der III. Furchung begann und etwa 1/2 Stunde vor derselben wieder verschwand. Dies kann man wohl ebenso wie bei der II. Kernteilung als elektromotorischen Ausdruck der III. Kernteilung ansehen, die der III. Furchung vorangeht.

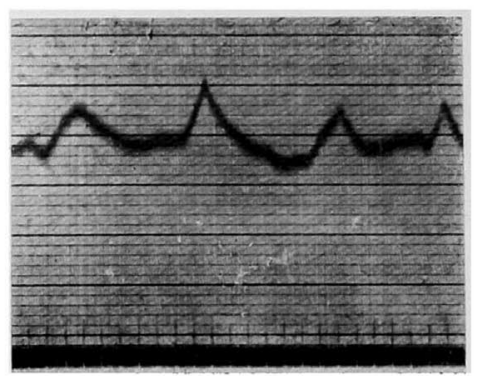

a

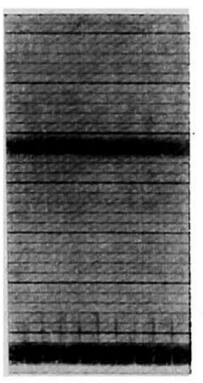

b

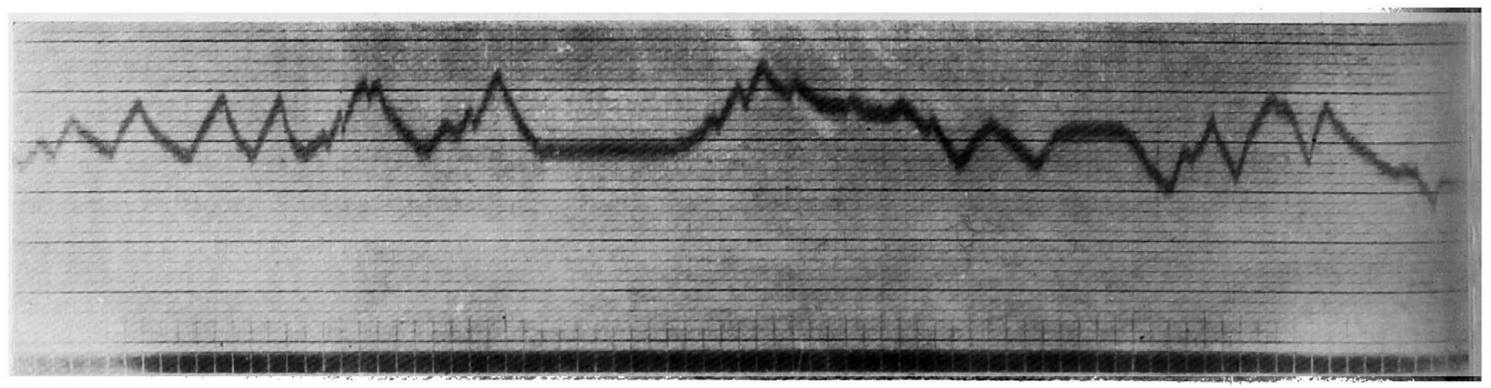

c

Abb. 1. Potentialschwankungen vor und während der zweiten Furchung (Ableitung von Aktionsströmen von beiden Polen). a Potentialschwankungen 50 Minuten vor Beginn der zweiten Furchung; b Effektlosigkeit der Ableitung 10 Minuten vor Beginn der zweiten Furchung; c Potentialschwankungen während der zweiten Furchung. Zeitmarken 3 Sek. 


\section{Bei der III. Furchung...}

Etwa 2-2 $\frac{1}{2}$ Stunden nach Beginn der II. Furchung begaun die III.-te. Das zeitliche Verhalten der III. Furchenbildung war fast gleich wie bei den vorangehenden 2 Furchungen. Gleichzeitig mit Beginn der III. Furchung setzten Potentialschwankungen ein, die bei der Furchenbildung in der animalen Hemisphäre deutlich und frequent wurden, in der vegetativen Hemisphäre langsam und schwach blieben. Beim ersteren Fall war der animale $\mathrm{Pol}$ elektronegativ gegenüber dem vegetativen, beim letzteren umgekehrt. Diese Potentialschwankungen während der III. Furchung waren grösser als die während der entsprechenden III. Kernteilung und als die während der II. Furchung. Einige Zeit vor der IV. Furchung, wo die III. Furchung schon den vegetativen Pol erreicht hatte und vom Ei kein Aktionsstrom mehr ableitbar war, traten auch Potentialschwankungen plötzlich auf, die schon vor der IV. Furchung aufhörten. Diese Schwankungen sind wohl das elektromotorische Zeichen der IV. Kernteilung, die die IV. Furchung des Eidotters und des Protoplasmas anbahnt.

\section{Zusammenfassung.}

1. Von der Oberfäche des Tritoneies sind während seiner einzelnen Furchungen mono- oder diphasische Aktionsströme ableitbar; in ihrem Auftreten gibt es zwei Zeitabschnitte: (a) Etwa 1 Stunde vor den einzelnen Furchungen beginnen die Aktionsströme und hören etwa 1/2 Stunde vor denselben auf. (b) Mit Beginn der einzelnen Furchungen an der Eioberfäche beginmen die Aktionsströme und verschwinden, wenn die Furchenbildung den vegetativen Pol erreicht. Die Aktionsströme bei (b) sind der elektromotorische Ausdruck der Teilung des Protoplasmas und des Eidotters, diejenigen bei (a) sind das elektromotorische Zeichen der den einzelnen Teilungen des Eidotters und des Protoplasmas vorangehenden entsprechenden Kernteilung.

2. Die Potentialdifferen\% bei den einzelnen Furchungen ist grösser als die bei den entsprechenden Kernteilungen.

3. Bei den einzelnen Kernteilungen wird der animale Pol elektro. negativ gegenüber dem vegetativen; bei den einzelnen Furchungen wird zuerst der animale $\mathrm{Pol}$ elektronegativ gegenüber dem vegetativen, später aber ungekehrt.

4. Die Potentialdifferenz wird von Furchung zu Furchung allmählich grösser. 
Zum Schluss möchte ich Herrn Prof. Dr. B. Hasama herzlich danken, der mir gefällig den Saitengalvanometer im hiesigen pharmakologischen Institut zur Verfügung gestellt hat.

\section{Literatur.}

1) O. Jordan, The habits and development of the newt (Diemictylus viridescens). Journ. of Morphol. Vol. 8, S. 318. 1893.

2) Id a $\mathrm{H}$. $\mathrm{Hyde}$, Differences in electrical potential in developing eggs. Americ. Journ. Physiol. Bd. 12, S. 241. 1904.

3) Dorfman, Electrical polarity of the amphibian egg and its reversal through fertilization. Protoplasma. Bd. 21, S. 245. 1934. 